\title{
Stress response recruits the hippocampal endocannabinoid system for the modulation of fear memory
}

\author{
Lucas de Oliveira Alvares, ${ }^{1,2,4}$ Douglas Senna Engelke, ${ }^{1,4}$ Felipe Diehl, ${ }^{1,2}$ \\ Robson Scheffer-Teixeira, ${ }^{1}$ Josué Haubrich, ${ }^{1}$ Lindsey de Freitas Cassini, ${ }^{1}$ \\ Victor Alejandro Molina, ${ }^{2,3}$ and Jorge Alberto Quillfeldt ${ }^{1,2,5}$
}

${ }^{1}$ Laboratório de Psicobiologia e Neurocomputação, Departamento de Biofísica, Instituto de Biociências, Universidade Federal do Rio Grande do Sul, CEP 91.501-970, Porto Alegre, Rio Grande do Sul, Brazil; ${ }^{2}$ Programa de Pós-Graduação em Neurociências, Instituto de Ciências Básicas da Saúde, Universidade Federal do Rio Grande do Sul-Porto Alegre, CEP 90.046-900, Porto Alegre, Rio Grande do Sul, Brazil; ${ }^{3}$ Departamento de Farmacologia, Facultad de Ciencias Quimicas, Universidad Nacional de Córdoba, Haya de la Torre y Medina Allende, Ciudad Universitaria 5000, Córdoba, Argentina

\begin{abstract}
The modulation of memory processes is one of the several functions of the endocannabinoid system (ECS) in the brain, with $\mathrm{CB}$ 1 receptors highly expressed in areas such as the dorsal hippocampus. Experimental evidence suggested an important role of the ECS in aversively motivated memories. Similarly, glucocorticoids released in response to stress exposure also modulates memory formation, and both stress and dexamethasone activate the ECS. Here, we investigate the interaction between the ECS and glucocorticoids in the hippocampus in the modulation of fear memory consolidation. Two protocols with different shock intensities were used in order to control the level of aversiveness. Local infusion of AM251 into the hippocampus immediately after training was amnestic in the strong, but not in the weak protocol. Moreover, AM251 was amnestic in animals stressed 0, but not 30-min prior to the weak protocol, reverting the stress-induced facilitatory effect. Finally, intrahippocampal AM251 infusion reduced memory in animals that received dexamethasone immediately, but not 30 min before training. These results are (1) consistent with the view that the dorsal hippocampus ECS is activated on demand, in a rapid and short-lived fashion in order to modulate the consolidation of an aversive memory, and (2) show that this recruitment seems to be mediated by glucocorticoids, either in the hippocampus or in other brain regions functionally associated with the hippocampus.
\end{abstract}

In the last decade, growing evidence supports the role of the endocannabinoid system (ECS) as a memory modulator, particularly in mammal brain structures (Davies et al. 2002; Lutz 2007; Viveros et al. 2007; Heifets and Castillo 2009). The involvement of this modulatory system in areas such as the dorsal hippocampus or the basolateral amygdala (BLA) is not only corroborated by receptor density studies (Mackie 2005; Marsicano and Kuner 2008), but is also consistent with several pharmacological results, usually obtained in behavioral tasks that are both aversive and hippocampus dependent. For instance, CB1 antagonists were shown to impair consolidation (de Oliveira Alvares et al. 2005) and extinction (Suzuki et al. 2004; Pamplona and Takahashi 2006; Niyuhire et al. 2007), while having the opposite effect on memory retrieval (de Oliveira Alvares et al. 2008a) and reconsolidation (de Oliveira Alvares et al. 2008b). The same results may not be observable when the administration is performed systemically or under a different experimental protocol (Suzuki et al. 2004; Pamplona and Takahashi 2006; Yim et al. 2008). The ECS may be acting both at the cellular and the systems level, being involved in memory processes that depend on protein synthesis (de Oliveira Alvares et al. 2008b; Heifets and Castillo 2009; Puighermanal et al. 2009) and, on the other side of the spectrum,

\footnotetext{
${ }^{4}$ These authors contributed equally to this work.

${ }^{5}$ Corresponding author.

E-mail quillfe@ufrgs.br; fax 55(51)3308-7003.

Article is online at http://www.learnmem.org/cgi/doi/10.1101//m.1721010.
}

is considered a good spike timing/brain oscillations coordinator candidate (Robbe et al. 2006). Although the role of the ECS in long-term memory modulation is better documented for aversive tasks, this system does not appear to be involved in less-aversive memories (Hölter et al. 2005; de Oliveira Alvares et al. 2006; Pamplona and Takahashi 2006; Niyuhire et al. 2007).

Among the several different stress hormones released after an aversive learning paradigm, glucocorticoids (GC) are noticeable for their broad functional and temporal range of effects (Joëls 2008; Joëls et al. 2009). The hippocampus is one of the memoryrelated targets of these actions, being specifically susceptible to uncontrollable stress through a pathway that involves the amygdala (Kim and Diamond 2002; Akirav and Richter-Levin 2006; Malin and McGaugh 2006). Several convergent studies show that either corticosterone, or the synthetic glucocorticoid dexamethasone, or stress promote the enhancement of memory consolidation (Roozendaal and McGaugh 1996; Roozendaal et al. 1999) while impairing retrieval (de Quervain et al. 1998; Roozendaal et al. 2003). Moreover, recent studies have shown that stress exposure or glucocorticoid administration impair reconsolidation (Maroun and Akirav 2008; Wang et al. 2008) and influence extinction (Cai et al. 2006; Yang et al. 2006) despite some contradictory results (Maroun and Akirav 2008). It is quite uncommon for a drug to exhibit a functional profile structured like that exhibited by agents acting at the ECS, i.e., enhancing consolidation while impairing retrieval, as well as causing exactly opposite effects upon reconsolidation and extinction. 
The parallels between endocannabinoids and glucocorticoids acting upon different phases of memory processing suggest an interaction between both systems, as already shown in other brain areas (Di et al. 2003, 2005; Steiner and Wotjak 2008). A recent report suggests that CB1-mediated signaling in the basolateral amygdala is critically modulated by GCs in emotional memory consolidation (Campolongo et al. 2009). In addition, there are studies showing that both stress and dexamethasone administration increased the level of both endocannabinoids anandamide and 2-arachidonylglycerol (Di et al. 2003, 2005; Hohmann et al. 2005). The aim of the current study was to evaluate the interaction between the ECS and glucocorticoids in the modulation of memory consolidation using the contextual fear conditioning (CFC) paradigm, a well known hippocampus-dependent learning task (Kim and Fanselow 1992). Moreover, the training protocol used allows one to control the strength of the aversive stimulus applied.

\section{Results}

Experiment 1: The endocannabinoid system is recruited only with strong fear training

In this first series of experiments, we study whether the ECS is required for memory consolidation when the training used a mild footshock $(0.3 \mathrm{~mA})$ or when the training used a strong footshock $(0.7 \mathrm{~mA})$. With the strong footshock training, there was a significant difference between the AM251 and the control groups (Fig. 1A, $P<0.001, t$-test). In contrast, such an effect was not evident with the weak footshock protocol (Fig. 1B, $P=0.750$, $t$-test). The fact that the AM251 disruptive effect was absent using the weak protocol suggests that only high levels of aversive status may be able to recruit the hippocampal endocannabinoid system in order to modulate contextual fear memory.

\section{Experiment 2: Prior stress recruits the hippocampal endocannabinoid system for the modulation of fear memory consolidation}

Based on the findings obtained in the experiment 1 , we next asked whether increasing the aversiveness from another source prior to the weak training protocol would be able to render memory consolidation sensitive to the interference induced by the CB1 antagonist. Figure 2 shows the freezing behavior performed during the test by animals that received a bilateral intrahippocampal infusion of AM251 or its vehicle after the CFC weak training, and with a previous stress session in a different context at two different times. The two-way ANOVA revealed a significant effect for AM251 $\left(F_{(1,35)}=11.145, P=0.002\right)$ and an interaction between stress-0-min $\times \operatorname{AM} 251\left(F_{(1,35)}=4.556, P=0.040\right)$. There was a significant difference between the AM251 and its DMSO control for 0 min (Tukey post-hoc test, $P<0.001$ ), but not for 30 -min pretraining stress (idem, $P=0.419$ ); also, the AM251 group for 0 min pre-training stress differed significantly from the $30 \mathrm{~min}$ case (idem, $P=0.021$ ). These results showed that a prior stressful experience strengthens the memory consolidation of a weak conditioning protocol. Moreover, this rapid memory-enhancing effect elicited by stress is modulated by the activation of the hippocampal ECS.

\section{Experiment 3: Glucocorticoids interact with the hippocampal endocannabinoid signaling to modulate fear memory consolidation}

In the following experiment, we investigated whether glucocorticoids recruit the hippocampal ECS to influence memory
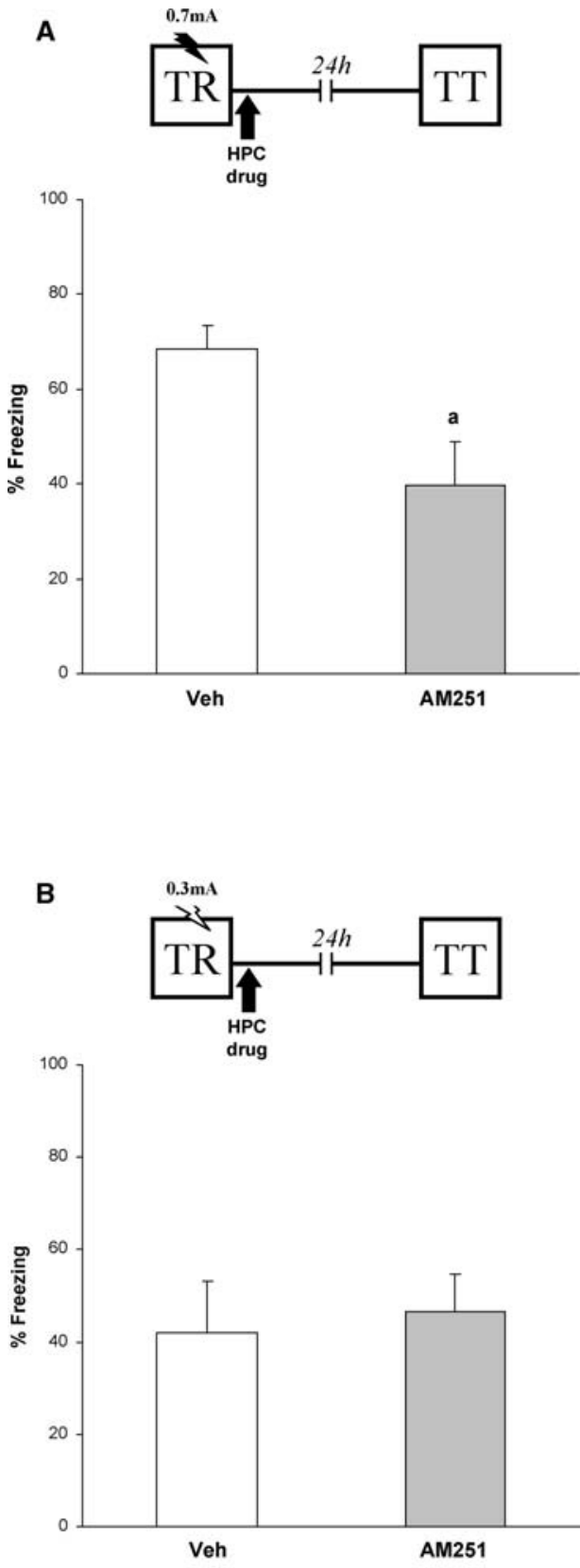

Figure 1. Effect of bilateral intrahippocampal infusion of AM251 or its vehicle (veh: PBS with $8 \%$ DMSO) upon percentual freezing time in a test session performed $24 \mathrm{~h}$ after a contextual fear conditioning (CFC) training session under $(A)$ a strong $(0.7 \mathrm{~mA})$ or $(B)$ a weak $(0.3 \mathrm{~mA})$ footshock. The diagram above each histogram depicts the corresponding experimental design. Data expressed as mean \pm SEM of percent of freezing time in a 5-min test session. (a) Significantly different from the control group ( $P<0.001, t$-test), with $n=11$ and 9 , respectively. In Figure 2 (bottom panel) there were no significant differences between groups $(P=0.750, t$-test), with $n=8$ and 8 , respectively.

consolidation following the weak footshock protocol. To this end, we studied the effect of a systemic injection of an exogenous synthetic glucocorticoid injected at 0 or $30 \mathrm{~min}$ prior to the CFC weak training. The goal of this experiment was to mimic the effect of the stressful experience observed in experiment 2. For the groups injected with dexamethasone immediately before 


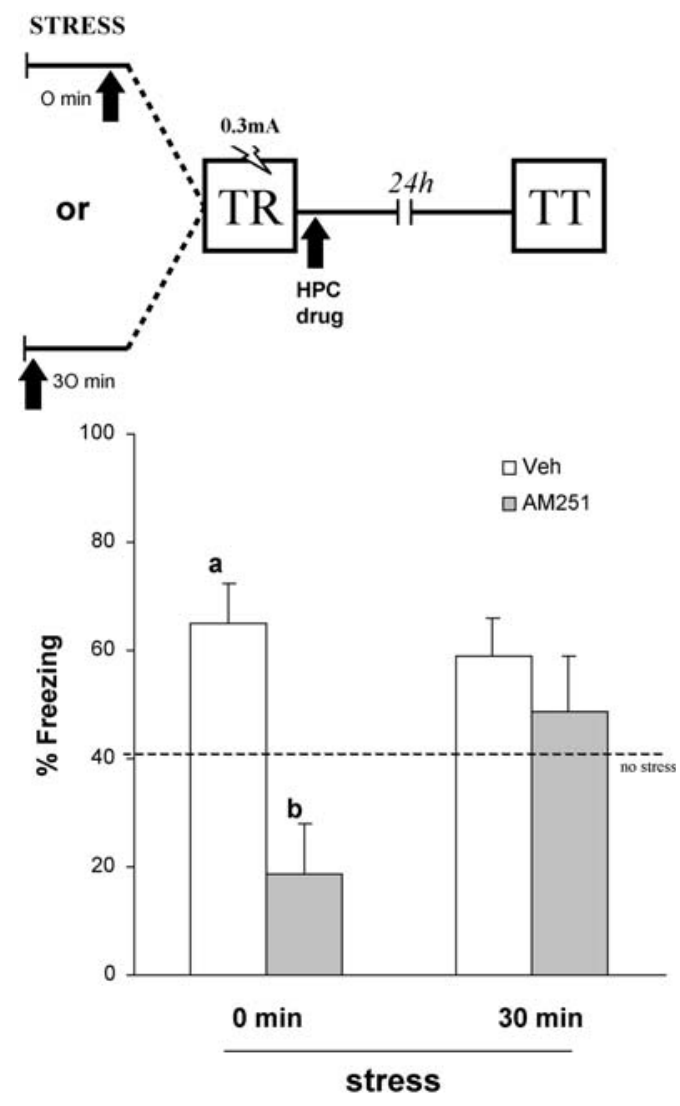

Figure 2. Time-dependent differential responses (percentual freezing) to bilateral intrahippocampal infusion of AM251 or its vehicle (veh: PBS with $8 \%$ DMSO), in a test session performed $24 \mathrm{~h}$ after a CFC training session under both a weak $(0.3 \mathrm{~mA})$ footshock and after a previous stress session in a different context, at different times. The diagram above the histogram depicts the corresponding experimental design. Data expressed as mean \pm SEM of percentual freezing time in a 5 -min test session. Dashed line: average value of percent freezing for a group not submitted to stress, shown for illustrative reasons only. Horizontal axis: time of stress before CFC training. Two-way ANOVA indicates a significant effect of treatment (but not of time), and an interaction between treatment and time: (a) significant difference between treatment groups for this time $(P<0.001$, Tukey post-hoc test); (b) significantly different from the correspondent AM251-infused group stressed at $30 \mathrm{~min}(P=$ 0.021 , Tukey post-hoc test). $n=11,10,10$, and 8 , respectively.

training (Fig. 3A), two-way ANOVA indicated a significant effect of AM251 $\left(F_{(1,31)}=5.464, P=0.026\right)$, and an interaction between DEXA and AM251 groups $\left(F_{(1,31)}=13.554, P<0.001\right)$ : There was a significant difference between both DMSO groups (Tukey post-hoc test, $P=0.003$ ); and the AM251 group injected with DEXA was significantly different from its DMSO control (idem, $P<0.001)$. For the groups injected with dexamethasone $30 \mathrm{~min}$ before training (Fig. 3B), the two-way ANOVA indicates only a significant effect of DEXA $\left(F_{(1,35)}=17.051, P<0.001\right)$ : There was a significant difference between groups receiving DEXA or its vehicle for each intrahippocampal treatment, be it DMSO (Tukey post-hoc test, $P=0.003$ ) or AM251 (idem, $P=0.011$ ). These data showed that dexamethasone induced a long-lasting facilitatory effect upon memory consolidation, a potentiation that was blocked by AM251 infused into the hippocampus. These results suggest that the rapid memory-enhancing effect of a glucocorticoid upsurge is modulated by the activation of the ECS in the hippocampus. Thus, dexamethasone mimicked the facilitating influence of a pre-training stress session upon memory consolidation following the weak training protocol.

\section{Experiments 4 and 5: Effect of intrahippocampal dexamethasone on the hippocampal endocannabinoid} signaling on the modulation of fear memory consolidation In order to analyze whether the stress/glucocorticoid-inducing effect upon the hippocampal endocannabinoid system takes place in the hippocampus, we performed two additional experiments. In experiment 4 , we investigated whether dexamethasone directly infused into the CA1 area immediately after the CFC training with a weak footshock could lead to the same enhancing effect observed in experiment 3: There was no statistically significant increase in the percent freezing following the weak footshock protocol (Fig. 4). Drug concentrations were similar to those used elsewhere (Abrahám et al. 1996; Ferreira et al. 2000; Di et al. 2003) and pretraining infusions were also ineffective (data not shown). Thus, intrahippocampal dexamethasone infusion failed to mimic the facilitating influence of a pretraining stress session or systemic dexamethasone on fear memory consolidation following the weak training protocol.

In additional experiments we evaluated the effect of mifepristone (RU486), a glucocorticoid cytoplasmatic receptor antagonist, in concentrations used earlier (Calfa et al. 2007), directly infused into the CA1 area immediately before the CFC training with a strong footshock. Since there is no membrane/ rapid/nongenomic GC receptor (mGCR) antagonist available yet (Di et al. 2005), we checked whether at least the slow, genomic-mediated GC hippocampal receptors might contribute to the observed enhancing effect induced by the strong shock (see Fig. 1A). The findings of this experiment showed comparable levels of freezing among the different groups $(P=0.947$, one-way ANOVA), indicating that the intrahippocampal infusion of this drug at two different doses does not affect fear memory consolidation. As in experiment 1, all groups were run in parallel, at the same time, and the means were: Vehicle: $39.2 \pm 5.5 \mathrm{sec}$; Mifepristone $6 \mathrm{ng} / \mu \mathrm{L}$ : $42.4 \pm 8.1 \mathrm{sec}$; Mifepristone $60 \mathrm{ng} / \mu \mathrm{L}$ : $39.9 \pm 10.0 \sec (n=9,8$, and 5, respectively). The smaller freezing time of the control group compared with that in Figure 1A (with same shock intensity) might be due to natural variations among samples, since the experiments were performed in different months of the year.

\section{Discussion}

The current study examined the role of the hippocampal ECS in the modulation of memory consolidation induced by different footshock intensities during fear conditioning. The amnestic effect of the CB1 antagonist AM251 on consolidation following the strong footshock protocol (Fig. 1A) is consistent with previous data from our group using both the CFC and the step-down inhibitory avoidance tasks (de Oliveira Alvares et al. 2005, 2006, $2008 \mathrm{a}, \mathrm{b})$. In contrast, such effect was absent in animals subjected to weak footshock training (Fig. 1B). These findings suggest that a strong emotionally arousing experience is a necessary condition for the involvement of the hippocampal ECS on fear memory consolidation.

The view that hippocampal ECS requires some level of aversiveness in order to be recruited is also supported by studies showing that memory motivated by mildly aversive tasks, such as the open field habituation, systematically fails to respond to CB1 agents (de Oliveira Alvares et al. 2005, 2008a). Although the generality of this supposition might be disputed (see, e.g., 
A
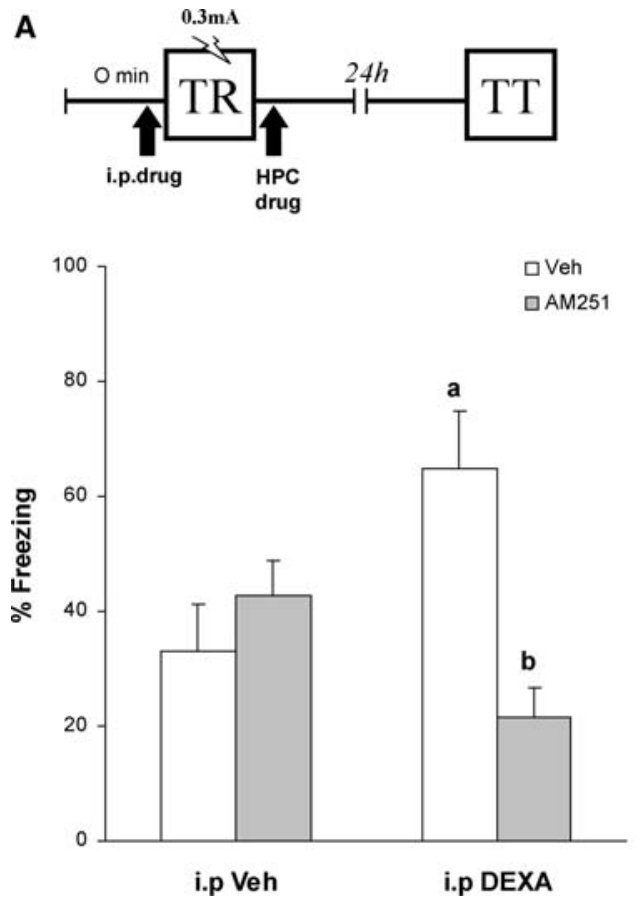

B
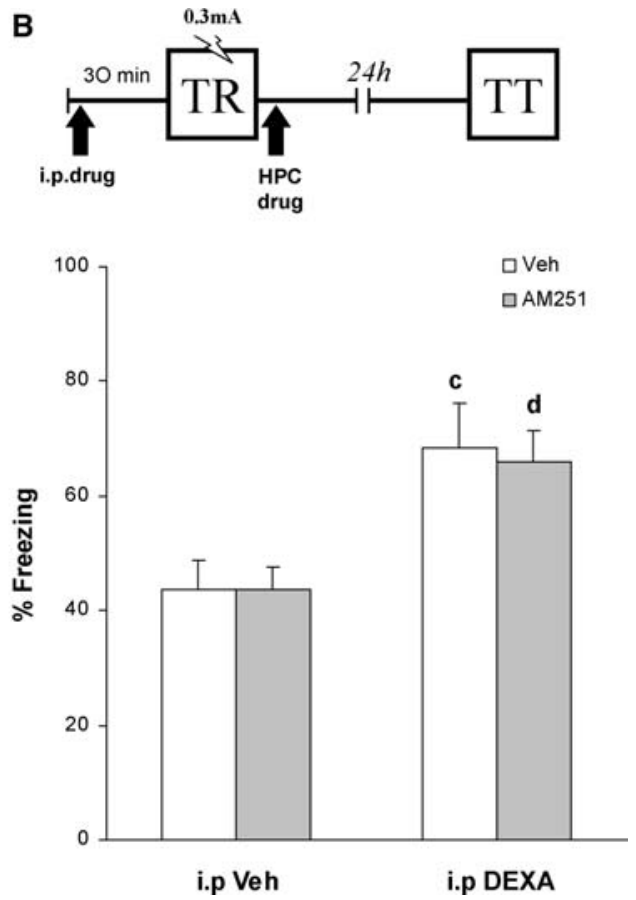

Figure 3. Interdependency between intrahippocampal treatment (AM251 or its vehicle) and dexamethasone systemically infused (A) immediately $(0 \mathrm{~min})$ or $(B) 30 \mathrm{~min}$ before the CFC training session under a weak $(0.3 \mathrm{~mA})$ footshock. The diagram above each histogram depicts the corresponding experimental design. Data expressed as mean \pm SEM of percentual freezing time in a 5-min test session performed $24 \mathrm{~h}$ after the CFC training session. Horizontal axis: The two intrahippocampal infusion groups (Veh: PBS with 8\% DMSO; AM251:5.5 ng/side) are grouped according to the systemic treatment-first the control group (i.p. Veh: saline $\pm 2.5 \%$ ethanol) and, next, the dexamethasone group (i.p. DEXA: $0.01 \mathrm{mg} / \mathrm{Kg}$ ). In $A$ the two-way ANOVA indicates a significant effect of intrahippocampal, but not of systemic treatment, and an interaction between these treatments: (a) significantly different from the intrahippocampal vehicle-injected of the i.p. Veh systemic group $(P=0.003$, Tukey post-hoc test); (b) significantly different from the correspondent vehicle-injected group of the same systemic (i.p. DEXA) group $(P<0.001$, Tukey post-hoc test). $n=9,7,10$, and 9 , respectively. In $B$, the two-way ANOVA indicates a significant effect of systemic (but neither of intrahippocampal treatment nor an interaction between the two treatments): There is a significant difference between systemic groups for each intrahippocampal treatment, (c) Veh $(P=0.003$, Tukey post-hoc test) or (d) AM251 ( $P=0.011$, Tukey post-hoc test), with $n=11,9,10$, and 9, respectively.

Kamprath et al. 2006; Jacob et al. 2009), it seems consistent with the literature indicating that ECS involvement is task-specific (de Oliveira Alvares et al. 2005; Hölter et al. 2005; Niyuhire et al. 2007). Recently, Kamprath et al. (2009) have demonstrated a dependency of endocannabinoid action on the intensity of the footshock used in a fear-conditioning task that associates tone response with previous shock treatment.

It is widely known that stressful events, such as an aversive learning paradigm, or the release of hormones functionally associated with threatening events, play a critical role in memory processes (Roozendaal and McGaugh 1996; de Quervain et al. 1998; Roozendaal et al. 1999, 2003; Okuda et al. 2004). It is noticeable how the effects of these stimuli resemble the endocannabinoid system on its influence on different memory phases (Suzuki et al. 2004; de Oliveira Alvares et al. 2005, 2008a,b; Cai et al. 2006; Pamplona and Takahashi 2006; Yang et al. 2006; Niyuhire et al. 2007; Maroun and Akirav 2008; Wang et al. 2008). Actually, the release of endocannabinoids such as anandamide and 2-AG have been demonstrated in the hippocampus (Kamprath et al. 2006), the amygdala (Marsicano et al. 2002), and other brain structures, such as the periaqueductal gray matter (Hohmann et al. 2005) and the midbrain (Di et al. 2005), always in response to an aversive situation. Another suggestive clue comes from the fact that cannabinoids can influence synaptic events taking place in areas such as the hippocampus, particularly after an aversive stimulation (Wilson et al. 2001; Carlson et al. 2002).

Consonant with this view, the present study shows that intrahippocampus infusion of AM251 blocked the memory- enhancing effect of both pre-training treatments, a single stress session (Fig. 2) or a single dexamethasone injection (Fig. 3A), suggesting an activation of the hippocampal ECS by the glucocorticoid system. Endocannabinoids would, in turn, suppress the local interneuronal GABAergic control (Katona et al. 1999; Wilson and Nicoll 2002) and, through this mechanism, disinhibit the memory trace building excitatory pathway as we have suggested elsewhere (de Oliveira Alvares et al. 2005, 2006).

In the last few years, it became clear that the HypothalamusPituitary-Adrenal (HPA) axis activity is controlled by an endocannabinoid tonus (Cota 2008; Steiner and Wotjak 2008); more specifically, subcortical brain areas expressed CB1 receptors that seem to be involved in stress-induced GC release (Steiner et al. 2008). The existence of a two-way interdependence between endocannabinoid and GC systems began to be uncovered after the demonstration of the existence of rapid/nongenomic/ membrane-bound glucocorticoid receptors (Tasker et al. 2006); and corticosterone, an endogenous GC released by the adrenal cortex in response to a stressful stimulus, can act upon several targets in the brain after freely crossing the blood-brain barrier (Joëls 2008). It was suggested that the fact that this effect takes place in a few minutes was in conflict with the well-known slow, genomic effects mediated by the two citoplasmatic types of GC receptors. This evidence led to the proposal of a fast, functional G-protein membrane-bound receptor (mGCR), first shown in the hypothalamic PVN area (Di et al. 2003, 2005): Postsynaptic mGCRs in parvocellular neurons can promote a fast feedback inhibition of further hormone release, a mechanism mediated 


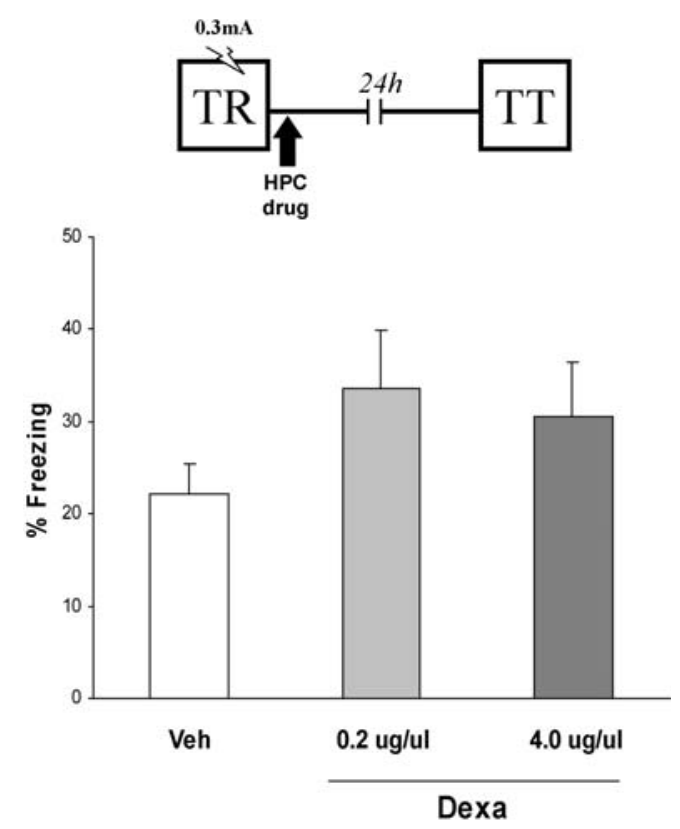

Figure 4. Effect of dexamethasone (Dexa: 0.2 and $4 \mu \mathrm{g} / \mu \mathrm{L}$ ) or its vehicle (Veh: PBS) directly infused into the dorsal hippocampus (area CA1) immediately after the CFC training under a weak $(0.3 \mathrm{~mA})$ shock. Data expressed as mean \pm SEM of percentual freezing time in the 5-min test session performed $24 \mathrm{~h}$ after the CFC training session. There were no significant differences between the groups $(P=0.332$, one-way ANOVA). $n=12,13$, and 14 , respectively.

by the production and release of endocannabinoids acting presynaptically to reduce glutamate release (Di et al. 2003). Similarly, GCs can reduce glutamate and increase GABA release in magnocellular neurons through endocannabinoids that will retrogradely act upon two different cell types (Di et al. 2005), using two divergent G-protein pathways (Di et al. 2009). This putative fast-signaling receptor might explain the different results we have observed between the two time points selected ( 0 or 30 prior to training) for stress exposure (Fig. 2), or dexamethasone administration (Fig. 3A,B), suggesting that the intrahippocampal infusion of the CB1 antagonist disrupted memory consolidation only at the beginning of the GC system activation.

In order to conclude whether the GC-dependent hippocampal endocannabinoid recruitment takes place in the hippocampus itself, we performed experiments 4 and 5 . These results, however, are inconclusive, and the "link" may still be in the hippocampus-possibly mediated by the putative, fast mGCRs-or in another brain structure. In this regard, two recent reports showed that local AM251 infusion into the BLA may interfere with the memory-enhancing effect of stress or GCs (Campolongo et al. 2009; Ganon-Elazar and Akirav 2009). In the first study, the memory-enhancing effect of GCs was attributed to an ECSmediated disinhibitory influence on noradrenaline release, a mechanism that facilitated the formation of the aversive memory trace (Campolongo et al. 2009; Hill and McEwen 2009). In the second work, intra-BLA AM251 disrupted an avoidance memory extinction and the agonist WIN55,212-2, administered either into the BLA or systemically, modulated the behavioral enhancement effect of stress, prompting a small increase in plasma corticosterone levels (Ganon-Elazar and Akirav 2009). Thus, not only the dorsal hippocampus and the basolateral amygdala may share an analogous mechanism, but they might even be functionally connected in order to modulate a cognitive process such as the formation of a new contextual fear memory trace (Akirav et al. 2001; Kim and Diamond 2002; Akirav and Richter-Levin 2006; Malin and McGaugh 2006). Further experiments are necessary to investigate such a possibility.

The dexamethasone i.p. dose selected in the present study enhances memory consolidation in a long-lasting way (Fig. 3A,B). Despite the fact that this synthetic glucocorticoid has a different time profile as compared with corticosterone (Bohus and De Kloet 1981), experiment 3 was able to effectively mimic the stress situation, again supporting the idea that the stress-induced enhancing effect involves the recruitment of hippocampal endocannabinoids (Fig. 3A), either directly or through an external relay as mentioned above. Furthermore, this ECS recruitment appears to vanish $30 \mathrm{~min}$ after dexamethasone administration (Fig. 3B), similar to the effect we observed when stress exposure was performed $30 \mathrm{~min}$ prior to the CFC training session (Fig. 2). This was also consistent with evidence showing that endocannabinoids are rapidly released on demand and have a brief half-life of circa 5 min (Di Marzo et al. 1994, 2005).

Since the stress-enhancing effect took place only when presented immediately before (or during) training, a time course of action for the stress-released endogenous agent that mediates the phenomenon is suggested. The fact that the effect is no longer evident when animals are subjected to the environmental demand 30 min prior to training is consistent with the time course of several stress hormones (Joëls 2008). In this line of reasoning, corticosterone may be a good candidate, even considering the fact that its hippocampal peak takes place $30 \mathrm{~min}$ after stress (Pfaff et al. 1971; Joëls 2008) and similar times may be expected for nearby anatomical targets. Thus, its receptor-mediated effects, both rapid and/or slow, might be induced early in the time curve, and the receptor activation/inactivation cycle might be completed well before the GCs have reached their maximum levels.

In summary, our results suggest that the activation of the ECS in the hippocampus requires a certain level of aversiveness or emotional status to exert its modulatory role on fear memory consolidation. This negative emotional state may be provided (1) by the task stimulus itself (e.g., a strong shock; Fig. 1A), or (2) by a previous stress session (Fig. 2), or, alternatively, (3) by a hormone functionally associated with a stressful stimulus, such as the glucocorticoids (Fig. 3A). Based on these findings we propose that glucocorticoids may be the putative endogenous mediators of this aversive-dependent hippocampal ECS recruitment, despite the fact that this functional link may be taking place in the same or in adjacent brain structures. To our knowledge, this is the first demonstration that the hippocampal ECS can functionally interact with glucocorticoids in order to modulate the formation of a contextual fear memory.

\section{Materials and Methods}

\section{Animals}

Two-hundred and thirty-one male Wistar rats (age 2-3 mo, weight 250-320 g) from our breeding colony were used in these experiments. Animals were housed in plastic cages, four to five in a cage, under a 12-h light/dark cycle and at a constant temperature of $24 \pm 1^{\circ} \mathrm{C}$, with water and food ad libitum.

\section{Stereotaxic surgery and cannulae placement}

All animals were anesthetized by a mixture of ketamine and xylazine (i.p., 75 and $10 \mathrm{mg} / \mathrm{kg}$, respectively) and bilaterally implanted with a 27 -gauge guide cannulae aimed at AP $-4.2 \mathrm{~mm}$ (from bregma), LL $\pm 3.0 \mathrm{~mm}$, DV $1.8 \mathrm{~mm}$, just $1.0 \mathrm{~mm}$ above the CA1 area of the dorsal hippocampus (according to Paxinos and Watson 2007). After a 1-wk recovery from surgery, animals were submitted to the behavioral procedures. Following the 
behavioral experiments, all subjects were sacrificed and their brains dissected and preserved in $10 \%$ formaldehyde to verify for cannulae position under low magnification (Fig. 5): 210 out of 231 animals had correct cannulae placements, so their data were included in the statistical analysis.

\section{Contextual fear conditioning (CFC)}

The conditioning chamber consisted of an illuminated Plexiglas box $(25.0 \times 25.0-\mathrm{cm}$ grid of parallel $0.1-\mathrm{cm}$ caliber stainless steel bars spaced $1.0 \mathrm{~cm}$ apart). In the conditioning session (training), rats were placed in the chamber for 3 min for habituation, and only after this, they received two 2 -sec footshocks, either of 0.3 or $0.7 \mathrm{~mA}$, separated by a $30-\mathrm{sec}$ interval. Before returning to their home cages, animals were kept in the conditioning environment for an additional minute. Twenty-four hours later, all animals were tested for $5 \mathrm{~min}$ in the same context.

\section{Stress vs. glucocorticoid injection}

The stress session consisted of receiving two 1-mA inescapable footshocks in a different context $(10 \times 10-\mathrm{cm}$ plastic box, grid floor), delivered $10 \mathrm{sec}$ after being put there. Both stress exposure or dexamethasone i.p administration. $(0.01 \mathrm{mg} / \mathrm{kg})$ were either applied 30 min or immediately before training in the CFC task. In order to avoid any association with the grid floor itself, the very few animals (less than five) that presented freezing behavior during the 3-min habituation phase of the conditioning session (before receiving the conditioning shocks) were excluded from the analyses.

\section{Intrahippocampally infused drugs}

At the time of infusion, a 30-gauge infusion needle was fitted into the guide cannulae, with its tip protruding $1.0 \mathrm{~mm}$ beyond the guide cannula end and aimed at the pyramidal cell layer of CA1 of the dorsal hippocampus (see Fig. 5). A volume of $0.5 \mu \mathrm{L}$ was bilaterally infused at a slow rate $(20 \mu \mathrm{L} / \mathrm{h})$ and the needle was removed only after another additional $30 \mathrm{sec}$. In experiments $1-3$ animals were divided into two groups, each receiving one of the following drugs: AM251, a selective CB1 antagonist $(5.5 \mathrm{ng} /$ side; Tocris Cookson, Inc.), or its vehicle (phosphatebuffered saline with $8 \%$ dimethylsulfoxide). Vehicle composition and the doses/concentrations used were selected based on previous experiments from our laboratory (de Oliveira Alvares et al. 2006, 2008b). Dexamethasone (0.2 and $4 \mu \mathrm{g} / \mu \mathrm{L})$ and the GCR antagonist mifespristone/RU486 (Sigma; 6 and $60 \mathrm{ng} / \mu \mathrm{L}$ ) for the intrahippocampal infusions were dissolved in the same vehicle as described above for AM251.

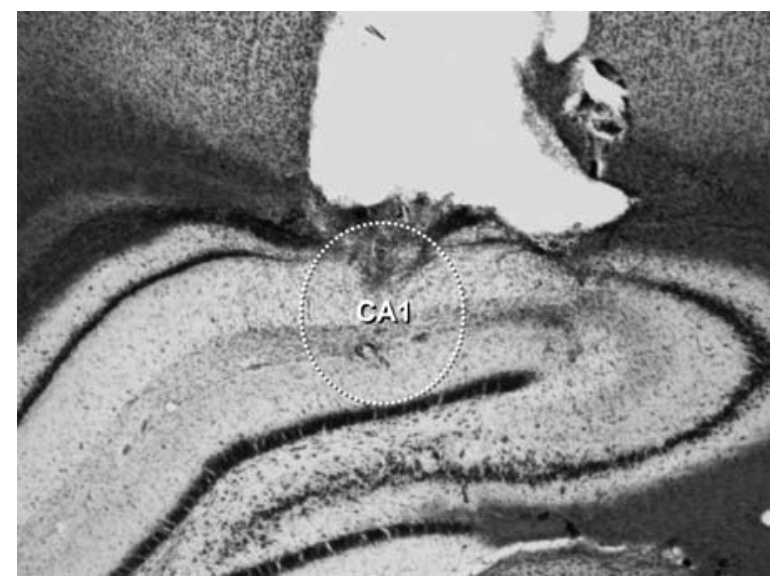

Figure 5. Typical acceptable needle placement, aimed at the CA1 region (according to Paxinos and Watson 2007) of the rat dorsal hippocampus (formol thionine technique).

\section{Experimental design}

\section{Experiment 1}

Animals were trained in the CFC with either a strong $(0.7 \mathrm{~mA})$ or a weak $(0.3 \mathrm{~mA})$ footshock; immediately after the training session, rats received the intrahippocampal infusion of AM251 (5.5 ng/ side) or its vehicle (PBS + 8\% DMSO), and the test was performed $24 \mathrm{~h}$ later (see the diagram above Fig. 1A,B).

\section{Experiment 2}

Animals were submitted to a stressful event in a different context at different time points, either 0 or $30 \mathrm{~min}$ prior to the training session, and then trained in the weak shock protocol $(0.3 \mathrm{~mA})$; immediately after training, animals received locally in the dorsal hippocampus either AM251 or its vehicle (as in experiment 1); test was performed $24 \mathrm{~h}$ later (see the diagram above Fig. 2).

\section{Experiment 3}

Animals were injected i.p. with dexamethasone (DEXA, $0.01 \mathrm{mg} /$ $\mathrm{Kg})$ or its vehicle $(\mathrm{ETOH}, \mathrm{PBS}+2.5 \%$ ethanol) at different time points, either 0 or $30 \mathrm{~min}$ prior to the training session, and then trained using the weak shock protocol $(0.3 \mathrm{~mA})$; immediately after training, animals received locally in the dorsal hippocampus either AM251 or its vehicle (as in experiment 1); test was performed $24 \mathrm{~h}$ later (see diagram above Fig. 3A,B).

\section{Experiment 4}

Experiment 4 was similar to experiment 1, with dexamethasone $(0.2$ and $4 \mu \mathrm{g} / \mu \mathrm{L})$ directly infused into the dorsal hippocampus (area CA1) after the training session with the weak $(0.3 \mathrm{~mA})$ shock (see diagram above Fig. 4).

\section{Experiment 5}

Experiment 5 was also similar to experiment 1, with mifepristone ( 6 and $60 \mathrm{ng} / \mu \mathrm{L}$ ) directly infused into the dorsal hippocampus (area CA1) before the training session with the strong $(0.7 \mathrm{~mA})$ shock.

\section{Statistical analysis}

Since CFC data (percent freezing) in all experimental groups have both reached $P>0.200$ in the Kolmogorov-Smirnov test with Lilliefors' correction and passed the Equal Variance test, normality and homocedasticity were ensured and only parametric tests were used (data expressed as mean \pm SEM). Differences between groups receiving intrahippocampal post-training infusions of AM251 or its vehicle after a weak or a strong footshock were identified by $t$-test for independent samples (experiment 1); when more groups were involved, one-way (experiments 4 and 5) or two-way (experiments 2 and 3) ANOVAs was used and differences sorted by Tukey all-pairwise multiple comparison post-hoc test. Statistical analysis of the behavioral data was limited to the 210 out of 231 animals with correct cannulae placements (Fig. 5), and only $P$ of $<0.05$ were considered significant.

\section{Ethical aspects}

All experimental procedures in living animals (rats) were performed in strict accordance to the recommendations of the Brazilian Society for Neurosciences (SBNeC), the Brazilian College of Animal Experimentation (SBCAL), and the International Brain Research Organization (IBRO), being previously approved by our Institutional (UFRGS) Committee for Ethics in Research, meaning that they are in compliance with the U.S. National Institutes of Health Guide for Care and Use of Laboratory Animals (publication no. 85-23, revised in 1985), the European Communities Council Directive of 24 November 1986 (86/609/EEC), and the Brazilian law (Federal Law no 11.794/ 2008). Every effort was made to minimize the number of animals used and their suffering. 


\section{Acknowledgments}

This research was supported by fellowships and grants from the CAPES (MEC), CNPq (MCT), PROPESQ (UFRGS), International Foundation for Science (IFS, Stockholm), and FINEP ("Rede Instituto Brasileiro de Neurociências," IBN-Net, No. 01.06.0842-00). We acknowledge Zelma Regina V. de Almeida for her kind technical assistance. We also thank Drs. Carla Dalmaz and Sidia Callegari Jacques for their helpful comments and suggestions.

\section{References}

Abrahám I, Juhász G, Kékesi KA, Kovács KJ. 1996. Effect of intrahippocampal dexamethasone on the levels of amino acid transmitters and neuronal excitability. Brain Res 733: 56-63.

Akirav I, Richter-Levin G. 2006. Factors that determine the non-linear amygdala influence on hippocampus-dependent memory. Dose Response 4: 22-37.

Akirav I, Sandi C, Richter-Levin G. 2001. Differential activation of hippocampus and amygdala following spatial learning under stress. Eur J Neurosci 14: 719-725.

Bohus B, de Kloet ER. 1981. Adrenal steroids and extinction behavior: Antagonism by progesterone, deoxycorticosterone and dexamethasone of a specific effect of corticosterone. Life Sci 28: 433-440.

Cai WH, Blundell J, Han J, Greene RW, Powell CM. 2006. Postreactivation glucocorticoids impair recall of established fear memory. J Neurosci 26: 9560-9566.

Calfa G, Bussolino D, Molina VA. 2007. Involvement of the lateral septum and the ventral Hippocampus in the emotional sequelae induced by social defeat: Role of glucocorticoid receptors. Behav Brain Res 181: $23-34$.

Campolongo P, Roozendaal B, Trezza V, Hauer D, Schelling G, McGaugh JL, Cuomo V. 2009. Endocannabinoids in the rat basolateral amygdala enhance memory consolidation and enable glucocorticoid modulation of memory. Proc Natl Acad Sci 106: 4888-4893.

Carlson G, Wang Y, Alger BE. 2002. Endocannabinoids facilitate the induction of LTP in the hippocampus. Nat Neurosci 5: 723-724.

Cota D. 2008. The role of the endocannabinoid system in the regulation of hypothalamic-pituitary-adrenal axis activity. J Neuroendocrinol 20: $35-38$.

Davies SN, Pertwee RG, Riedel G. 2002. Functions of cannabinoid receptors in the hippocampus. Neuropharmacology 42: 993-1007.

de Oliveira Alvares L, de Oliveira LF, Camboim C, Diehl F, Genro BP, Lanziotti VB, Quillfeldt JA. 2005. Amnestic effect of intrahippocampal AM251, a CB1-selective blocker, in the inhibitory avoidance, but not in the open field habituation task, in rats. Neurobiol Learn Mem 83: 119-124.

de Oliveira Alvares L, Genro BP, Vaz Breda R, Pedroso MF, Da Costa JC, Quillfeldt JA. 2006. AM251, a selective antagonist of the CB1 receptor, inhibits the induction of long-term potentiation and induces retrograde amnesia in rats. Brain Res 1075: 60-67.

de Oliveira Alvares L, Genro BP, Diehl F, Quillfeldt JA. 2008a. Differential role of the hippocampal endocannabinoid system in the memory consolidation and retrieval mechanisms. Neurobiol Learn Mem 90: 1-9.

de Oliveira Alvares L, Pasqualini Genro B, Diehl F, Molina VA, Quillfeldt JA. 2008b. Opposite action of hippocampal CB1 receptors in memory reconsolidation and extinction. Neuroscience 154: 1648-1655.

de Quervain DJ, Roozendaal B, McGaugh JL. 1998. Stress and glucocorticoids impair retrieval of long-term spatial memory. Nature 394: $787-790$

Di S, Malcher-Lopes R, Halmos KC, Tasker JG. 2003. Nongenomic glucocorticoid inhibition via endocannabinoid release in the hypothalamus: A fast feedback mechanism. J Neurosci 23: 4850-4857.

Di S, Malcher-Lopes R, Marcheselli VL, Bazan NG, Tasker JG. 2005. Rapid glucocorticoid-mediated endocannabinoid release and opposing regulation of glutamate and $\gamma$-aminobutyric acid inputs to hypothalamic magnocellular neurons. Endocrinology 146: 4292-4301.

Di S, Maxson MM, Franco A, Tasker JG. 2009. Glucocorticoids regulate glutamate and GABA synapse-specific retrograde transmission via divergent nongenomic signaling pathways. J Neurosci 29: 393-401.

Di Marzo V, Fontana A, Cadas H, Schinelli S, Cimino G, Schwartz JC, Piomelli D. 1994. Formation and inactivation of endogenous cannabinoid anandamide in central neurons. Nature 372: 686-691.

Di Marzo V, De Petrocellis L, Bisogno T. 2005. The biosynthesis, fate and pharmacological properties of endocannabinoids. Handb Exp Pharmacol 168: 147-185.
Ferreira VM, Takahashi RN, Morato GS. 2000. Dexamethasone reverses the ethanol-induced anxiolytic effect in rats. Pharmacol Biochem Behav 66: 585-590.

Ganon-Elazar E, Akirav I. 2009. Cannabinoid receptor activation in the basolateral amygdala blocks the effects of stress on the conditioning and extinction of inhibitory avoidance. J Neurosci 29: 11078-11088.

Heifets BD, Castillo PE. 2009. Endocannabinoid signaling and long-term synaptic plasticity. Annu Rev Physiol 71: 283-306.

Hill MN, McEwen BS. 2009. Endocannabinoids: The silent partner of glucocorticoids in the synapse. Proc Natl Acad Sci 106: 4579-4580.

Hohmann AG, Suplita RL, Bolton NM, Neely MH, Fegley D, Mangieri R, Krey JF, Walker JM, Holmes PV, Crystal JD, et al. 2005. An endocannabinoid mechanism for stress-induced analgesia. Nature 435: 1108-1112.

Hölter SM, Kallnik M, Wurst W, Marsicano G, Lutz B, Wotjak CT. 2005. Cannabinoid CB1 receptor is dispensable for memory extinction in an appetitively motivated learning task. Eur J Pharmacol 510: 69-74.

Jacob W, Yassouridis A, Marsicano G, Monory K, Lutz B, Wotjak CT. 2009. Endocannabinoids render exploratory behaviour largely independent of the test aversiveness: Role of glutamatergic transmission. Genes Brain Behav doi: 10.1111/j.1601-183X.2009.00512.x.

Joëls M. 2008. Functional actions of corticosteroids in the hippocampus. Eur J Pharmacol 583: 312-321.

Joëls M, Krugers HJ, Lucassen PJ, Karst H. 2009. Corticosteroid effects on cellular physiology of limbic cells. Brain Res doi: 10.1016/ j.brainres.2009.03.036.

Kamprath K, Marsicano G, Tang J, Monory K, Bisogno T, Di Marzo V, Lutz B, Wotjak CT. 2006. Cannabinoid CB1 receptor mediates fear extinction via habituation-like processes. J Neurosci 26: 6677-6686.

Kamprath K, Plendl W, Marsicano G, Deussing JM, Wurst W, Lutz B, Wotjak CT. 2009. Endocannabinoids mediate acute fear adaptation via glutamatergic neurons independently of corticotropin-releasing hormone signaling. Genes Brain Behav 8: 203-211.

Katona I, Sperlagh B, Sik A, Kafalvi A, Vizi ES, Mackie K, Freund TF. 1999. Presynaptically located CB1 cannabinoid receptors regulate GABA release from axonal Terminals of specific hippocampal interneurons. J Neurosci 19: 4544-4558.

Kim JJ, Fanselow MS. 1992. Modality-specific retrograde amnesia of fear. Science 256: $675-677$

Kim JJ, Diamond DM. 2002. The stressed hippocampus, synaptic plasticity and lost memories. Nat Rev Neurosci 3: 453-462.

Lutz B. 2007. The endocannabinoid system and extinction learning. $\mathrm{Mol}$ Neurobiol 36: 92-101.

Mackie K. 2005. Distribution of cannabinoid receptors in the central and peripheral nervous system. Handb Exp Pharmacol 168: 299-325.

Malin EL, McGaugh JL. 2006. Differential involvement of the hippocampus, anterior cingulate cortex, and basolateral amygdala in memory for context and footshock. Proc Natl Acad Sci 103: 1959-1963.

Maroun M, Akirav I. 2008. Arousal and stress effects on consolidation and reconsolidation of recognition memory. Neuropsychopharmacology 33: 394-405.

Marsicano G, Kuner R. 2008. Anatomical distribution of receptors, ligands and enzymes in the brain and in the spinal cord: Circuitries and neurochemistry. In Cannabinoids and the Brain (ed. A Kofälvi), pp. 161-201. Springer, Heidelberg, Germany.

Marsicano G, Wotjak CT, Azad SC, Bisogno T, Rammes G, Cascio MG, Hermann H, Tang J, Hofmann C, Zieglgänsberger W, et al. 2002. The endogenous cannabinoid system controls extinction of aversive memories. Nature 418: 530-534.

Niyuhire F, Varvel SA, Thorpe AJ, Stokes RJ, Wiley JL, Lichtman AH. 2007 The disruptive effects of the CB1 receptor antagonist rimonabant on extinction learning in mice are task-specific. Psychopharmacology 191: $223-231$.

Okuda S, Roozendaal B, McGaugh JL. 2004. Glucocorticoid effects on object recognition memory require training-associated emotional arousal. Proc Natl Acad Sci 101: 853-858.

Pamplona FA, Takahashi RN. 2006. WIN 55212-2 impairs contextual fear conditioning through the activation of CB1 cannabinoid receptors. Neurosci Lett 397: 88-92.

Paxinos G, Watson C. 2007. The rat brain in stereotaxic coordinates. Academic Press, San Diego, CA.

Pfaff DW, Silva MT, Weiss JM. 1971. Telemetered recording of hormone effects on hippocampal neurons. Science 172: 394-395.

Puighermanal E, Marsicano G, Busquets-Garcia A, Lutz B, Maldonado R, Ozaita A. 2009. Cannabinoid modulation of hippocampal long-term memory is mediated by mTOR signaling. Nat Neurosci 12: $1152-1158$.

Robbe D, Montgomery SM, Thome A, Rueda-Orozco PE, McNaughton BL, Buzsaki G. 2006. Cannabinoids reveal importance of spike 
timing coordination in hippocampal function. Nat Neurosci 9: $1526-1533$

Roozendaal B, McGaugh JL. 1996. Amygdaloid nuclei lesions differentially affect glucocorticoid-induced memory enhancement in an inhibitory avoidance task. Neurobiol Learn Mem 65: 1-8.

Roozendaal B, Nguyen BT, Power AE, McGaugh JL. 1999. Basolateral amygdala noradrenergic influence enables enhancement of memory consolidation induced by hippocampal glucocorticoid receptor activation. Proc Natl Acad Sci 96: 11642-11647.

Roozendaal B, Griffith QK, Buranday J, De Quervain DJ, McGaugh JL. 2003. The hippocampus mediates glucocorticoid-induced impairment of spatial memory retrieval: Dependence on the basolateral amygdala. Proc Natl Acad Sci 100: 1328-1333.

Steiner MA, Wotjak CT. 2008. Role of the endocannabinoid system in regulation of the hypothalaic-pituitary-adrenocortical axis. Progress Brain Res 170: $397-432$.

Steiner MA, Marsicano G, Wotjak CT, Lutz B. 2008. Conditional cannabinoid receptor type 1 mutants reveal neuron subpopulation-specific effects on behavioral and neuroendocrine stress responses. Psychoneuroendocrinology 33: 1165-1170.

Suzuki A, Josselyn SA, Frankland PW, Masushige S, Silva AJ, Kida S. 2004 Memory reconsolidation and extinction have distinct temporal and biochemical signatures. J Neurosci 24: 4787-4795.
Tasker JG, Di S, Malcher-Lopes R. 2006. Minireview: Rapid glucocorticoid signaling via membrane-associated receptors. Endocrinology 147: $5549-5556$.

Viveros MP, Marco EM, Llorente R, López-Gallardo M. 2007. Endocannabinoid system and synaptic plasticity: Implications for emotional responses. Neural Plast doi: 10.1155/2007/52908.

Wang XY, Zhao M, Ghitza UE, Li YQ Lu L. 2008. Stress impairs reconsolidation of drug memory via glucocorticoid receptors in the basolateral amygdala. J Neurosci 28: 5602-5610.

Wilson RI, Nicoll RA. 2002. Endocannabinoid signaling in the brain. Science 296: $678-682$.

Wilson RI, Kunos G, Nicoll RA. 2001. Presynaptic specificity of endocannabinoid signaling in the hippocampus. Neuron 31: 453-462.

Yang YL, Chao PK, Lu KT. 2006. Systemic and intra-amygdala administration of glucocorticoid agonist and antagonist modulate extinction of conditioned fear. Neuropsychopharmacology 31: 912-924. Yim TT, Hong NS, Ejaredar M, McKenna JE, McDonald RJ. 2008. Post-training CB1 cannabinoid receptor agonist activation disrupts long-term consolidation of spatial memories in the hippocampus. Neuroscience 151: 929-936.

Received December 11, 2009; accepted in revised form January 27, 2010. 


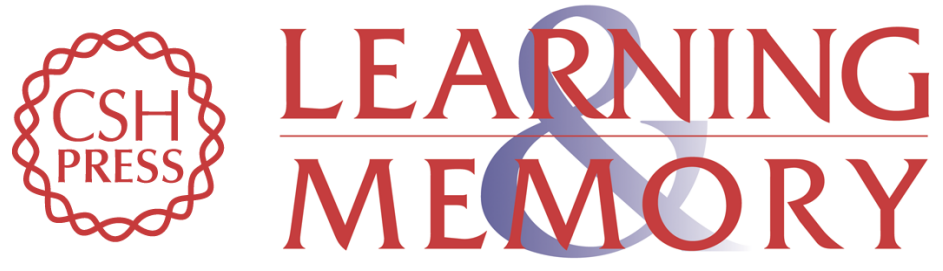

\section{Stress response recruits the hippocampal endocannabinoid system for the modulation of fear memory}

Lucas de Oliveira Alvares, Douglas Senna Engelke, Felipe Diehl, et al.

Learn. Mem. 2010, 17:

Access the most recent version at doi:10.1101//m.1721010

References This article cites 55 articles, 17 of which can be accessed free at:

http://learnmem.cshlp.org/content/17/4/202.full.html\#ref-list-1

License

Email Alerting Receive free email alerts when new articles cite this article - sign up in the box at the Service top right corner of the article or click here. 